\title{
Differential palliative care issues in patients with primary and secondary brain tumours
}

\author{
Christoph Ostgathe • Jan Gaertner • Maren Kotterba • \\ Sebastian Klein • Gabriele Lindena • \\ Friedemann Nauck $\cdot$ Lukas Radbruch . \\ Raymond Voltz • \\ For the Hospice and Palliative Care Evaluation (HOPE) \\ Working Group in Germany
}

Received: 29 January 2009 / Accepted: 20 August 2009/Published online: 8 September 2009

(C) Springer-Verlag 2009

\begin{abstract}
Introduction Cancer may affect the central nervous system either by primary or secondary brain tumours (PBT/SBT). Specific needs of these patients and special requirements of services caring for them have not been adequately addressed so far.

Methods Since 1999, an annual, 3-month census is being conducted in different palliative care settings throughout Germany. Pooled data from the years 2002-2005 were analysed to test for differences between patients with PBT, SBT and other palliative care patients (OP). Symptom
\end{abstract}

Lukas Radbruch and Raymond Voltz contributed equally to the manuscript.

C. Ostgathe · J. Gaertner $\cdot$ M. Kotterba $\cdot$ S. Klein $\cdot$ R. Voltz Centre for Palliative Medicine, University of Cologne,

Cologne, Germany

G. Lindena

Clinical Analysis, Research and Application, CLARA, Kleinmachnow, Germany

F. Nauck

Department of Palliative Medicine, University of Göttingen,

Göttingen, Germany

L. Radbruch

Department of Palliative Medicine, RWTH Aachen University,

Aachen, Germany

C. Ostgathe $(\bowtie)$

Zentrum für Palliativmedizin, Klinikum der Universität zu Köln,

Kerpenerstraße 62,

50924 Köln, Germany

e-mail: christoph.ostgathe@uk-koeln.de frequencies, ECOG status and nursing needs were tested for differences $\left(\chi^{2}\right)$. Free text entries for reasons for admission were recorded, categorised and analysed descriptively.

Results A total of 5,684 patients were documented (PBT $n=$ $153,2.7 \%$; SBT $n=661,11.9 \%$; OP $n=4,872,85.4 \%$ ). For patients with PBT, poor functional status and high need for nursing support was reported more frequently than for patients with SBT and OP. For patients with PBT/SBT physical symptoms (pain, nausea, vomiting, constipation and loss of appetite) were documented less frequently and in lower intensity than for OP. However, nursing, psychological, and social problems/symptoms were documented significantly more often and showed higher intensity, particularly the patients' need for support with the activities of daily living, disorientation/confusion of the patients and overburdening of the family. For $67 \%$ of all patients, a reason for admission was documented in free text. Main reasons were symptom control $(\mathrm{OP}>\mathrm{SBT}>\mathrm{PBT})$, social issues ( $\mathrm{PBT}>\mathrm{SBT}>\mathrm{OP}$ ), functional deficits ( $\mathrm{PBT}>\mathrm{SBT}>\mathrm{OP}$ ) and cognitive impairment (PBT/SBT $>\mathrm{OP}$ ). Distinct neurological deficits and problems were documented more often as secondary ICD-diagnoses than as a leading reason for admission.

Conclusion The specific palliative care problems and the rationales leading to health care utilisation point to the need for an appropriate interdisciplinary and multi-professional provision of care for patients with brain malignancies, with a particular view on the needs of the families and an early integration of social and psychological support.

Keywords Palliative care - Brain tumour - Brain metastases · Symptom $\cdot$ Documentation 
Table 1 Number of participating units and documented patients

\begin{tabular}{lrrrc}
\hline Year & 2002 & 2004 & 2005 & Number of patients \\
\hline Palliative care units & 54 & 68 & 53 & 4,182 \\
Hospices & 9 & 12 & 12 & 653 \\
General practitioners & - & 7 & 8 & 342 \\
Oncological units & 5 & 3 & 6 & 281 \\
Home care & - & 4 & 5 & 177 \\
Geriatric units & 2 & - & - & 49 \\
Documented pts & 1,567 & 2,214 & 1,903 & 5,684 \\
\hline
\end{tabular}

\section{Introduction}

Involvement of the central nervous system is common in patients with cancer. Secondary brain tumours develop in 20 $40 \%$ of systemic malignancies at some point during the illness [1]. Brain metastases significantly reduce life expectancy, with a mean survival of 1-6 months, depending on histology and applied therapies [2]. Primary brain tumours belong to the rarer neoplasms with less than $2 \%$ of the newly diagnosed cancers [3]. One fourth to one third of these are highly malignant gliomas with a median survival of 5-10 months [4, 5]. Primary or secondary brain tumours often lead to serious deterioration of neurological and cognitive functions [6]. Besides the existential distress, patients and their families suffer from fears that this condition may lead to a change or loss of individual control, cognition or consciousness, as well as the patients' personality.

Palliative care addresses symptoms and problems caused by progressive, life-limiting diseases irrespective of the underlying diagnosis [7]. Due to restricted options to limit the progression of the disease for the majority of patients with primary brain tumours, especially glioblastoma, or brain metastases, the management has to focus on the best possible palliation. To meet the specific needs of these patients appropriately, a more detailed evaluation of this specific group is required. Only little information on this topic is available [8,9], and it is neither known whether there are differences in the palliative care needs of patients with primary and secondary brain tumours nor whether differences to other palliative care patient groups exist.

In this study, we analysed prospectively collected core data sets of palliative care patients (1) to detect possible variations in palliative care problems in patients with malignancies in the central nervous system compared with other patients in palliative care and (2) to determine what the essential issues for services caring for this particular patient group are.

\section{Methods}

Since 1996, a standardised basic documentation tool (Hospice and Palliative care Evaluation (HOPE)) for palliative care patients has been developed, evaluated and amended accordingly by a multi-professional working group. Each year, German inpatient and outpatient hospice and palliative care services (Table 1) are recruited to participate in a 3-month census. The participants document up to 30 consecutive palliative care patients at admission to the service and at the time of discharge or death. The anonymised data can be submitted via use of an online database or a two-page paper questionnaire and is processed centrally.

For this study, data on age, gender, diagnosis, metastases, concomitant diseases, functional status, prevalence and intensity of symptoms as well as psychological, nursing and social problems were pooled (years 2002, 2004 and 2005 ${ }^{1}$ ) and analysed. Symptoms and problems were assessed by staff via an integrated symptom checklist (Table 2) using a 4-point grading scale $(0=$ none, $1=$ mild, $2=$ moderate, $3=$ severe). This symptom checklist was constructed as part of the documentation tool for a general palliative care population, serving to document a patient's status and not to reflect specific diagnoses (for example "feeling depressed" and not "depression"). Detailed information on the development of the documentation tool has been published previously $[10,11]$. HOPE was used in several epidemiological surveys in Germany [10, 12-14]. Further evaluation of the psychometric properties is currently being performed.

Patients with primary brain tumours were identified by the documented ICD10 Code and/or free text entries for the main diagnosis. Patients with secondary brain tumours could be identified as the core documentation included an item on the site of metastases. Differences between patients with primary and secondary brain tumours as well as between both groups and other patients without malignant affection of the central nervous system were analysed. Comparative analysis was performed for place of care, performance status according to the Eastern Cooperative

\footnotetext{
${ }^{1}$ Due to the fact that data analysis, feedback to the institutions and discussion about modification of the instrument lasted 15 months on average, no census was performed in 2003. Since 2005, the census has been repeated annually, as the infrastructure is now optimised and the instrument can be used as a standard documentation tool.
} 
Table 2 Symptoms and problems assessed with Hospice and Palliative care Evaluation (HOPE)

\begin{tabular}{ll}
\hline Physical & Pain, nausea, vomiting, dyspnoea, constipation, weakness, loss of appetite, tiredness \\
Nursing & Wound care, activities of daily living \\
Psychological & Feeling depressed, anxiety, emotional strain, disorientation/confusion \\
Social & Need for social support, overburden of family
\end{tabular}

Oncology Group (ECOG) [15], nursing levels, as well as for documented symptoms and problems. The nursing levels ${ }^{2}$ were graded according to the German legislation on long-term care in four different levels depending on type, frequency and duration of the need for nursing [16] (Table 3).

The programme packet SPSS 14.0 (SPSS Inc, Chicago, IL, USA) was used for statistical evaluation. Prevalence of symptoms and problems with higher intensity (moderate and severe) at the time of admission was determined. Group differences for these symptoms/problems, as well as for gender, place of care, levels of nursing care, ECOG were tested with chisquare, differences in age and length of stay and with ANOVA. For statistical significance we used $p<0.05$ in all tests.

The core data set includes free text entries for the reason for admission. These free text entries were recorded (e.g. pain, pain management, adaptation of analgesic regimen were coded as a subcategory pain) and categorised (e.g. pain and other symptoms were aggregated to a main category symptom management). As the instrument was not developed specifically for patients with brain malignancies, free text entries on reasons for admission were additionally scanned for specific neurological problems. This data was compared with neurological diagnoses documented as ICD-10 codes (seizures: G40-41; paresis: G 81-83/G 53.3; brain oedema: G93.6) to determine whether specific neurological problems were considered to be a reason for admission to palliative care. This data was evaluated descriptively only, as not for all patients a reason for admission was documented.

\section{Results}

A total of 5,684 palliative care patients were documented in the period under review. More than $50 \%$ of the German palliative care units $(2002,79$ units; 2004, 106; 2005,116) $[13,17]$ participated. The number of participating hospices ${ }^{3}$,

\footnotetext{
${ }^{2}$ According to the German long-term care legislation patients have to apply for a nursing level in order to receive financial aids. The grouping to a certain level implies a dedicated need for nursing support (higher level = higher need for nursing support (Table 3)) and aligns for a certain financial grant.

${ }^{3}$ In Germany, palliative care units are medical units, integrated into hospitals and have an average stay of 9 days. Hospices focus on nursing needs, are freestanding, generally patients remain there until they die. Funding for services also differs.
}

general practitioners, oncology wards, home care services and geriatric wards was considerably lower (Table 1).

A primary brain tumour (PBT) was the main diagnosis in 151 patients $(2.6 \%)$; 661 patients $(11.9 \%)$ with other primary cancers had brain metastases (secondary brain tumours (SBT)) at the time of admission. The most common sites of the primary were lung $(38.7 \%)$, breast $(19.5 \%)$ and gastrointestinal tract $(11.3 \%)$. No gender differences were detectable. Patients suffering from malignancies of the brain were significantly younger than other patients (PBT, 62.8 years \pm 14.8; SBT, 63.2 years \pm 12.3 ; other palliative care patients (OP), 68.4 years \pm 12.3 ; PBT/SBT, n.s.; PBT/OP, F $29.1 p<$ 0.001 ; SBT/OP, F $42.4 p<0.001$ ).

\section{Differential palliative care issues}

Functional status (ECOG) and need for nursing differed significantly. Patients with PBT and SBT showed more frequently a poor functional status (ECOG 3-4) compared with OP (Table 4). A high need for nursing support (level III) was documented more often for patients with PBT than for OP and patients with SBT (Table 4). The length of stay until discharge or death was significantly longer ( $\mathrm{F} 4.5 ; p=0.035)$ in patients with PBT (16.8 days \pm 16.1) compared with OP (14.3 days \pm 14.3$)$, but not in comparison with patients with SBT (14.5 days \pm 13.4$)$. In the period under review, significantly more patients with SBT died than OP or patients with PBT (Table 4).

The analysis of symptom/problem prevalences and intensities as documented in the symptom and problem checklist also showed significant differences. Most physical symptoms (except for weakness, tiredness and dyspnea) were documented less often with moderate to severe intensity in patients with $\mathrm{PBT} / \mathrm{SBT}$ compared with OP (Table 5). Need for support in the activities of daily living, symptoms of disorientation/confusion and problems with overburdening of the family were documented more often for patients with SBT and PBT (Table 5). Emotional strain and anxiety were more prevalent in patients with SBT compared with OP and patients with PBT (Table 5).

\section{Reason for admission}

In $67 \%(n=3,810)$ of all patients, a reason for admission was documented in free text. Here, mainly symptom control (PBT, 39.2\%; SBT, 57.4\%; OP 69,4\%), social issues (PBT, $27.8 \%$; SBT, $18.9 \%$; OP, 15.8\%), functional deficits (PBT, 
Table 3 Nursing levels according to the German long-term care legislation [16]

Level I

Support is necessary for at least two activities in the areas of personal hygiene, nutrition and mobility (at least once a day) as well as housekeeping (at least several times a week) with an overall average duration of at least $90 \mathrm{~min} /$ day

Level II Support is necessary at least three times a day with an overall average duration of at least $3 \mathrm{~h} /$ day

Level III

Level III + Support is necessary around the clock including nights with an overall average duration of at least $5 \mathrm{~h}$ /day Cases of hardship with exceptionally high need for support

14.4\%; SBT, 10.9\%; OP, 5.3\%) and cognitive impairment (PBT, 10.3\%; SBT, 10.5\%; OP, 3.5\%) were documented (Table 6). Distinct neurological deficits and problems were documented more often as secondary ICD-diagnoses than as a leading reason for admission (Table 6).

\section{Discussion}

Due to the lack of curative treatment options for many patients with primary brain tumours and their reduced life expectancy, good palliative care is essential [18]. For patients with cancer of other origin, the diagnosis of secondary brain tumours frequently determines the transition from a curative to a palliative situation [19]. Yet to date, there has been no systematic evaluation and comparison of specific palliative care problems and needs of patients with central nervous neoplasms within a palliative care population based on a broad, prospectively collected data pool.

The study presented here shows that symptoms and problems in patients with primary brain tumours differ significantly in prevalence and intensity from those of other palliative care patients. Patients with primary brain tumours had evidence of more advanced disease such as poorer performance status and higher levels of nursing support. However, physical symptoms, except weakness and tiredness, were documented less frequently and with lower intensity. Nursing problems and psychological problems showed little differences except for a predominance of disorientation and confusion in patients with primary brain tumours. The need for social support and overburdening of the family were significantly more often documented in patients with primary brain tumours.

Table 4 Comparison of characteristics (places of care, levels of nursing care and functional status) between the patients with primary brain tumours, secondary brain tumours and the other patients without malignant brain disease

\begin{tabular}{|c|c|c|c|c|c|c|c|c|c|}
\hline \multirow{3}{*}{$\begin{array}{l}\text { Group } \\
\text { Item }\end{array}$} & \multirow{2}{*}{\multicolumn{2}{|c|}{$\begin{array}{l}\text { Primary brain } \\
\text { tumour (PBT) } \\
N=151\end{array}$}} & & & & & Group differences & & \\
\hline & & & \multicolumn{2}{|c|}{$\begin{array}{l}\text { Secondary } \\
\text { brain tumour } \\
\text { (SBT) } N=661\end{array}$} & \multicolumn{2}{|c|}{$\begin{array}{l}\text { Other patients } \\
\text { (OP) } N=4,872\end{array}$} & \multirow[t]{2}{*}{$\mathrm{PBT} / \mathrm{SBT}$} & \multirow[t]{2}{*}{$\mathrm{PBT} / \mathrm{OP}$} & \multirow[t]{2}{*}{$\mathrm{SBT} / \mathrm{OP}$} \\
\hline & $N$ & $\%$ & $N$ & $\%$ & $N$ & $\%$ & & & \\
\hline \multicolumn{10}{|l|}{ Place of care } \\
\hline Palliative care unit & 103 & 68.2 & 480 & 72.6 & 3,599 & 73.9 & & & \\
\hline Hospice & 30 & 19.9 & 102 & 15.4 & 521 & 10.7 & & $p<0.001\left(\chi^{2} 12.6\right)$ & $p<0.001\left(\chi^{2} 13.1\right)$ \\
\hline General practitioner & 15 & 9.9 & 34 & 5.1 & 293 & 6 & $p=0.026\left(\chi^{2} 5.0\right)$ & $p=0.048\left(\chi^{2} 3.9\right)$ & \\
\hline Oncology & - & - & 15 & 2.3 & 266 & 5.5 & & & $p<0.001\left(\chi^{2} 12.3\right)$ \\
\hline Home care & 3 & 2 & 30 & 4.5 & 144 & 3 & & & $p=0.029\left(\chi^{2} 4.8\right)$ \\
\hline Geriatrics & - & - & - & - & 49 & 1 & & & \\
\hline \multicolumn{10}{|l|}{ Nursing levels } \\
\hline None & 35 & 23.2 & 224 & 33.9 & 2,096 & 43 & $p=0.011\left(\chi^{2} 6.5\right)$ & $p<0.001\left(\chi^{2} 23.6\right)$ & $p<0.001\left(\chi^{2} 19.9\right)$ \\
\hline Applied for & 35 & 23.2 & 148 & 22.4 & 854 & 17.5 & & & $p=0.002\left(\chi^{2} 9.3\right)$ \\
\hline Level I & 19 & 12.6 & 87 & 13.2 & 607 & 12.5 & & & \\
\hline Level II & 21 & 13.9 & 82 & 12.4 & 509 & 10.4 & & & \\
\hline Level III & 18 & 11.9 & 23 & 3.5 & 147 & 3.0 & $p<0.001\left(\chi^{2} 18.3\right)$ & $p<0.001\left(\chi^{2} 36.5\right)$ & \\
\hline Level III+ & - & - & 1 & 0.2 & 23 & 0.5 & & & \\
\hline ECOG 3 or 4 & 128 & 90.1 & 534 & 84.5 & 3,289 & 71.7 & & $p<0.001\left(\chi^{2} 23.3\right)$ & $p<0.001\left(\chi^{2} 46.3\right)$ \\
\hline Death & 62 & 44.0 & 349 & 55.2 & 2,176 & 47.3 & $p=0.015\left(\chi^{2} 5.9\right)$ & & $p<0.001\left(\chi^{2} 13.9\right)$ \\
\hline
\end{tabular}


Table 5 Comparison of prevalence of symptoms and other problems with higher intensity (moderate-severe) between the patients with primary brain tumours, secondary brain tumours and the other patients without malignant brain disease

\begin{tabular}{|c|c|c|c|c|c|c|c|c|c|}
\hline \multirow{3}{*}{$\begin{array}{l}\text { Group } \\
\text { Item }\end{array}$} & \multirow{2}{*}{\multicolumn{2}{|c|}{$\begin{array}{l}\text { Primary brain } \\
\text { tumour (PBT) } \\
N=151\end{array}$}} & \multirow{2}{*}{\multicolumn{2}{|c|}{$\begin{array}{l}\text { Secondary } \\
\text { brain tumour } \\
\text { (SBT) } N=661\end{array}$}} & & & Group differences & & \\
\hline & & & & & \multicolumn{2}{|c|}{$\begin{array}{l}\text { Other patients } \\
\text { (OP) } N=4872\end{array}$} & \multirow[t]{2}{*}{$\mathrm{PBT} / \mathrm{SBT}$} & \multirow[t]{2}{*}{$\mathrm{PBT} / \mathrm{OP}$} & \multirow[t]{2}{*}{$\mathrm{SBT} / \mathrm{OP}$} \\
\hline & $N$ & $\%$ & $N$ & $\%$ & $N$ & $\%$ & & & \\
\hline \multicolumn{10}{|l|}{ Physical symptoms } \\
\hline Pain & 43 & 29.5 & 289 & 44.1 & 2,590 & 53.5 & $p=0.001\left(\chi^{2} 10.9\right)$ & $p<0.001\left(\chi^{2} 33.8\right)$ & $p<0.001\left(\chi^{2} 20.9\right)$ \\
\hline Nausea & 16 & 10.7 & 125 & 19.2 & 1,350 & 28 & $p=0.015\left(\chi^{2} 5.9\right)$ & $p<0.001\left(\chi^{2} 21.6\right)$ & $p<0.001\left(\chi^{2} 22.6\right)$ \\
\hline Vomiting & 10 & 6.7 & 86 & 13.3 & 904 & 18.7 & $p=0.027\left(\chi^{2} 4.9\right)$ & $p<0.001\left(\chi^{2} 13.9\right)$ & $p=0.001\left(\chi^{2} 11.6\right)$ \\
\hline Dyspnoea & 3 & 2 & 191 & 29.1 & 1,365 & 28.3 & $p<0.001\left(\chi^{2} 48.4\right)$ & $p<0.001\left(\chi^{2} 49.7\right)$ & \\
\hline Constipation & 36 & 24.7 & 205 & 31.7 & 1,599 & 33.5 & & $p=0.026\left(\chi^{2} 4.9\right)$ & \\
\hline Weakness & 117 & 80.1 & 571 & 87.4 & 3,981 & 82.5 & $p=0.021\left(\chi^{2} 5.3\right)$ & & $p=0.002\left(\chi^{2} 9.9\right)$ \\
\hline Loss of appetite & 62 & 43.1 & 408 & 63.4 & 3,178 & 66.6 & $p<0.001\left(\chi^{2} 20.1\right)$ & $p<0.001\left(\chi^{2} 34.4\right)$ & \\
\hline Tiredness & 98 & 67.1 & 470 & 72.4 & 3,137 & 65.2 & & & $p<0.001\left(\chi^{2} 13.3\right)$ \\
\hline \multicolumn{10}{|l|}{ Nursing problems } \\
\hline Wound care & 24 & 16.4 & 135 & 21.1 & 986 & 20.2 & & & \\
\hline Activities of daily living & 139 & 92.7 & 553 & 85.1 & 3,375 & 70.6 & $p=0.014\left(\chi^{2} 6.0\right)$ & $p<0.001\left(\chi^{2} 34.5\right)$ & $p<0.001\left(\chi^{2} 59.7\right)$ \\
\hline \multicolumn{10}{|l|}{ Psychological problems } \\
\hline Feeling depressed & 45 & 32.1 & 223 & 35.1 & 1,494 & 31.5 & & & \\
\hline Anxiety & 46 & 32.2 & 267 & 41.7 & 1,765 & 37 & $p=0.035\left(\chi^{2} 4.4\right)$ & & $p=0.024\left(\chi^{2} 5.3\right)$ \\
\hline Emotional strain & 54 & 37.8 & 297 & 46.1 & 1,980 & 41.5 & & & $p=0.027\left(\chi^{2} 4.9\right)$ \\
\hline Disorientation/confusion & 70 & 50 & 226 & 35.1 & 669 & 14 & $p=0.001\left(\chi^{2} 10.8\right)$ & $p<0.001\left(\chi^{2} 137.5\right)$ & $p<0.001\left(\chi^{2} 182.9\right)$ \\
\hline \multicolumn{10}{|l|}{ Social problems } \\
\hline Need for social support & 84 & 58.7 & 343 & 54.9 & 2,032 & 43.2 & & $p<0.001\left(\chi^{2} 13.5\right)$ & $p<0.001\left(\chi^{2} 30.2\right)$ \\
\hline Overburden of family & 110 & 74.3 & 417 & 65.4 & 2,405 & 51.5 & $p=0.037\left(\chi^{2} 4.4\right)$ & $p<0.001\left(\chi^{2} 30.0\right)$ & $p<0.001\left(\chi^{2} 43.5\right)$ \\
\hline
\end{tabular}

The lower incidence of physical symptoms may be related to underrecognition and underassessment in those patients. This could be due to the difficult symptom assessment in cognitively impaired patients. In addition, the higher rate of symptomatic treatment with steroids could lead to alleviation of some symptoms such as loss of appetite in comparison to other patient groups [20].

The higher prevalence of social problems that was found in our study may be related to more advanced disease in primary brain tumour patients. However, the care for a loved one who suffers from disorientation and confusion may also overburden families and raise the need for social support. This has been identified as a major issue in families who care for patients with brain tumours, far more than in the care for the general palliative care population. Confusion impacts significantly on the social structures, as it aggravates high levels of distress in family members [9, 21,22]. This may lead to feelings of stress and problems of ill health in a majority of primary caretakers [9, 23]. Distress of family members may reflect the increase of

Table 6 Documented neurological problems of patients with primary and secondary brain tumours on admission

\begin{tabular}{|c|c|c|c|c|c|c|}
\hline & \multicolumn{2}{|c|}{ Primary brain tumour } & \multicolumn{2}{|c|}{ Secondary brain tumour } & \multicolumn{2}{|c|}{ Other patients } \\
\hline & $\begin{array}{l}\text { Diagnosis } \\
\text { (ICD) }\end{array}$ & $\begin{array}{l}\text { Reason for admission } \\
\text { (free text) }\end{array}$ & $\begin{array}{l}\text { Diagnosis } \\
\text { (ICD) }\end{array}$ & $\begin{array}{l}\text { Reason for admission } \\
\text { (free text) }\end{array}$ & $\begin{array}{l}\text { Diagnosis } \\
\text { (ICD) }\end{array}$ & $\begin{array}{l}\text { Reason for admission } \\
\text { (free text) }\end{array}$ \\
\hline Paresis & $34 \%$ & $2.6 \%$ & $10.3 \%$ & $2.1 \%$ & $2.9 \%$ & $0.3 \%$ \\
\hline Seizures & $18.6 \%$ & $8.2 \%$ & $6.1 \%$ & $3.1 \%$ & $0.5 \%$ & $0.1 \%$ \\
\hline Brain oedema & $6.2 \%$ & $5.2 \%$ & $1.3 \%$ & $1.7 \%$ & $0.1 \%$ & $0.1 \%$ \\
\hline
\end{tabular}


responsibilities with decreasing abilities of the patient and the uncertainty of the situation [24].

These issues are not merely specific to patients receiving palliative care and their families, but to the fact that the brain is affected by a malignant tumour. Therefore, the families' burden due to the patients' mental impairment and changed personality must be addressed appropriately already early in the course of the disease by all disciplines involved, e.g. neurooncologists, surgeons and radiotherapists.

Compared with patients with primary brain tumours, the group with secondary brain tumours showed a less reduced functional status and was assigned to lower levels of nursing care. Both parameters indicate less advanced disease than in patients with primary brain tumours, but still more advanced disease than in the other patients. Similarly, the differences in symptom scores between patients with primary brain tumours and other patients may be related to the general progression of the disease rather than to the fact that brain metastases have occurred. By nature of the disease, brain metastases frequently develop along with metastases at multiple sites as one of the signs of an overall progression of the disease. This is supported by the fact that in this group, significantly more patients died in the period under review.

By analysing the reasons for admission, we also wanted to explore what the essential needs are that services caring for these particular patient groups should provide. The emerging problems that lead to admission reflect some of the earlier mentioned issues. Although symptom management was the leading cause for admission in all groups, it was far less frequent in patients with primary and secondary brain malignancies than in other patients. Again, cognitive changes, functional deficits and social issues were identified far more commonly as the reason for service utilisation. Interestingly, even though specific neurological problems, such as paresis, seizures and brain oedema, were documented as ICD-diagnoses, they were rarely seen as the reason for admission by the documenting teams and thus not perceived as a specific focus for palliative care.

The described specific palliative care needs have to be taken into account when planning inpatient or outpatient care for these patient groups, but also for the implementation of a palliative care approach in neurological and oncological services.

More specifically, social or psychological support should be provided early and appropriately for patients with malignant cerebral involvement and their families to prevent family members from increasing distress and overburdening. Therefore, in services that care to a greater extent for palliative care patients with central malignancies, multi-professional structures with a focus on social work and psychology are of major importance. Offering respite care may be an additional and reasonable preventative intervention.
This study has some limitations. The categories presented in the documentation tool were developed with "general" palliative care patients. Issues specific for patients with brain tumours may therefore be underrepresented. As there are still only very few neurologically trained physicians in palliative care, some neurological problems may have been overlooked and the data presented may underestimate the real situation.

Furthermore, the documented symptoms and problems were assessed by staff and not by the patients themselves. As many of the documented patients in our study suffered from cognitive impairment, it may be assumed that the use of self-assessment would lead to a selection bias in favour of patients with better cognitive function. Even though there was no evaluation of the severity of cognitive impairment beyond staff assessment on a categorical scale, the routinely performed testing of the cognitive function of patients with brain malignancies at admission, for example with the Mini Mental State Examinations, may have strengthened the results of this study.

\section{Conclusion}

This study demonstrates for the first time on a broad, prospectively collected data pool that palliative care problems and needs in patients with primary and secondary brain tumours are significantly different compared with those of other patients. This information can be used for designing best possible palliative care. Specific palliative care problems and reasons for health care utilisation reveal the need for an appropriate provision of interdisciplinary and multi-professional care, with a particular view on the needs of the family caregivers and an early integration of social and psychological support.

Acknowledgements We would like to thank the physicians, nurses, social workers, psychologists and other staff members for the time and enthusiasm dedicated to data documentation. Additional thanks are directed to the principal investigators of the participating centres who participated in the development of HOPE, and to the German Association of Palliative Medicine, German Hospice and Palliative Association and the German Cancer Association for their support of HOPE.

\section{References}

1. Taillibert S, Delattre JY (2005) Palliative care in patients with brain metastases. Curr Opin Oncol 17:588-592

2. Pease NJ, Edwards A, Moss LJ (2005) Effectiveness of whole brain radiotherapy in the treatment of brain metastases: a systematic review. Palliat Med 19:288-299

3. Parkin DM, Pisani P, Ferlay J (1999) Global cancer statistics. CA Cancer J Clin 49:33-64 32 
4. Ohgaki H, Dessen P, Jourde B et al (2004) Genetic pathways to glioblastoma: a population-based study. Cancer Res 64:6892-6899

5. Smith JS, Jenkins RB (2000) Genetic alterations in adult diffuse glioma: occurrence, significance, and prognostic implications. Front Biosci 5:D213-231

6. Langer CJ, Mehta MP (2005) Current management of brain metastases, with a focus on systemic options. J Clin Oncol 23:6207-6219

7. WHO (2002) National cancer control programmes - policies and managerial guidelines. WHO, Geneva

8. Bausewein C, Hau P, Borasio GD et al (2003) How do patients with primary brain tumours die? Palliat Med 17:558-559

9. Catt S, Chalmers A, Fallowfield L (2008) Psychosocial and supportivecare needs in high-grade glioma. Lancet Oncol 9:884-891. doi:S14702045(08)70230-4[pii]10.1016/S1470-2045(08)70230-4

10. Radbruch L, Nauck F, Ostgathe C et al (2003) What are the problems in palliative care? Results from a representative survey. Support Care Cancer 11:442-451

11. Radbruch L, Ostgathe C, Elsner F et al (2004) What is the profile of palliative care in Germany. Results of a representative survey. Schmerz 18:179-188

12. Radbruch L, Nauck F, Fuchs M et al (2002) What is palliative care in Germany? Results from a representative survey. J Pain Symptom Manage 23:470-482

13. Radbruch L, Nauck F, Sabatowski R (2002) Germany: Cancer pain and palliative care - current situation. J Pain Symptom Manage 24:183-187

14. Nauck F, Ostgathe C, Klaschik E et al (2004) Drugs in palliative care: results from a representative survey in Germany. Palliat Med $18: 100-107$
15. Oken MM, Creech RH, Tormey DC et al (1982) Toxicity and response criteria of the Eastern Cooperative Oncology Group. Am J Clin Oncol 5:649-655

16. Busse R, Riesberg A (2004) Health care systems in transition: Germany. WHO Regional Office for Europe, on behalf of the European Observatory on Health Systems and Policies, Copenhagen

17. Schindler T (2006) Palliative care in Germany. Bundesgesundheitsblatt Gesundheitsforschung Gesundheitsschutz 49:1077-1086

18. Bausewein C, Borasio GD, Voltz R (2005) Brain tumours. In: Doyle D et al (eds) Oxford textbook of palliative medicine, 3rd edn. Oxford University Press, Oxford, pp 727-730

19. Ostgathe C, Voltz R (2008) Brain metastases. In: Walsh TD (ed) Palliative medicine, 1st edn. Mosby Inc. Philadelphia, pp 12401244

20. Oberndorfer S, Lindeck-Pozza E, Lahrmann H et al (2008) The end-of-life hospital setting in patients with glioblastoma. J Palliat Med 11:26-30

21. Namba M, Morita T, Imura $C$ et al (2007) Terminal delirium: families' experience. Palliat Med 21:587-594

22. Morita T, Akechi T, Ikenaga M et al (2007) Terminal delirium: recommendations from bereaved families' experiences. J Pain Symptom Manage 34(6):579-589

23. Faithfull S, Cook K, Lucas C (2005) Palliative care of patients with a primary malignant brain tumour: case review of service use and support provided. Palliat Med 19:545-550

24. Davies E, Clarke C, Hopkins A (1996) Malignant cerebral glioma-II: perspectives of patients and relatives on the value of radiotherapy. BMJ 313:1512-1516 